\title{
Kinetic Theory of Electrostatic Surface Waves in a Magnetized Plasma Slab
}

\author{
M.-J. Lee and Hee J. Lee* \\ Department of Physics, Hanyang University, Seoul 133-791, South Korea
}

\begin{abstract}
The dispersion relation of electrostatic surface waves propagating in a magnetized plasma slab is derived from Vlasov-Poisson equations under the specular reflection boundary condition. We consider the case that the static magnetic field is parallel to the plasma-vacuum interface and the surface wave propagates obliquely to the magnetic field on the parallel plane. We find that the specular reflection boundary conditions on the plasma slab-vacuum boundaries can be satisfied, even in a magnetized plasma, by simple extension of the electric potential into the vacuum region, due to the inherent symmetry of the distribution function. Utilizing an invariance property of the kinetic surface wave solution, it is shown that the two-mode structure of the surface wave yields the symmetric and anti-symmetric modes. The kinetic dispersion relation is checked against the dispersion relations obtained from fluid equations and shows complete agreement.
\end{abstract}

PACS numbers: 52.35.-g, 52.35. Hr, 52.25.Dg

Keywords: Plasma surface wave, magnetized, kinetic theory, plasma slab.

\section{INTRODUCTION}

Propagation of surface waves on the interface between a vacuum (or a dielectric) and plasmas has drawn much attention because of interest in bounded plasmas and their various technological applications $[1,2]$. Surface modes can be used for plasma diagnostics and surface-wave-produced plasmas are the subject of active investigation because such plasmas can be used in plasma processing [3, 4]. Furthermore surface waves are relevant to astrophysical problems in the magnetosphere and in the solar corona [5]. The solar atmosphere is highly structured, containing magnetic slabs and flux tubes. Surface waves are responsible for the heating of the solar corona. The Kelvin-Helmholtz instability is a surface wave instability. In the coupling of the ionosphere and the magnetosphere, surface waves are involved. Because of their importance, we have extensive list of papers for the investigation of the surface waves [6].

Surface modes on a magnetized plasma have been investigated in the context of fluid equations [7-10], but kinetic theory of surface waves in magnetized plasmas is rather few. In Ref. [11], cold plasma electrostatic surface wave dispersion relations are derived in the zero temperature limit of the kinetic dielectric tensor of an infinite magnetized plasma, but kinetic surface wave dispersion relations in hot magnetized plasmas are not derived. Earlier, the kinetic theory of surface waves in a semi-bounded unmagnetized moving plasma was investigated $[12,13]$.

Solving the Vlasov equation for a bounded plasma requires a kinematic boundary condition for the distribution function on the boundary. This condition is often taken to be the specular reflection condition [14], which is used here. In unmagnetized plasmas, the specular reflection boundary

*Address correspondence to this author at the Department of Physics, Hanyang University, Seoul 133-791, South Korea; Tel: +82-2-2220-1983; Fax: +82-2-2299-2028; E-mail: ychjlee@yahoo.com condition can be straightforwardly enforced by simple extension of the electric field components $[15,16]$. We find that, even in a magnetized plasma, the distribution function has a symmetry owing to which the specular reflection boundary condition is rendered to be satisfied by simple extension of the electromagnetic plasma fields.

In this work, we derive the dispersion relations of electrostatic surface waves propagating in magnetized slabs using the Vlasov-Poisson equations. Prior to this work, the dispersion relations of electromagnetic waves in unmagnetized slab were investigated [17]. A slab geometry entails an extra complication because we have two boundaries on which the specular reflection conditions have to be met. Earlier, Lee and Kim [18] investigated the electrostatic surface waves in magnetized slabs to derive the dispersion relations. In their work, the authors dealt with two cases of the magnetic field $\left(B_{0}\right)$ orientation; $B_{0}$ perpendicular and parallel to the plasma-vacuum interface [the surface waves propagate along the interface]. In the present work, we addresses the case that is not touched on by Lee and Kim; $B_{0}$ is parallel to the interface but the surface wave propagates obliquely to $B_{0}$ (Lee and Kim assumed that the wave propagates along $B_{0}$ ). In this oblique propagation, we find that an extra symmetry should be utilized when we use the invariance technique to solve the specular reflection boundary problem.

We checked our kinetic dispersion relations against the dispersion relations derived from fluid equations [19] and both results show complete agreement.

\section{SPECULAR REFLECTION BOUNDARY CONDITION}

We consider a magnetized plasma slab which occupies the region $(0<x<L)$ bounded by vacuum $(x<0$ and $x>L)$. The linearized Vlasov-Poisson equation for a 
species $\alpha$ ( $\alpha=\mathrm{e}, \mathrm{i}$; electron and ion), with charge $e_{\alpha}$ and mass $m_{\alpha}$, is

$\frac{\partial}{\partial t} f_{\alpha}(r, v, t)+v \cdot \frac{\partial f_{\alpha}}{\partial r}+\frac{e_{\alpha}}{m_{\alpha} c} v \times B_{0} \cdot \frac{\partial f_{\alpha}}{\partial v}=\frac{e_{\alpha}}{m_{\alpha}} \nabla \varphi(r, t) \cdot \frac{\partial f_{\alpha 0}}{\partial v}$.

where $f_{\alpha}$ is the perturbed distribution function of species $\alpha$ and $B_{0}$ is the static constant magnetic field $\left(=z B_{0}\right)$. The electric field $E=-\nabla \varphi$ satisfies the Poisson equation

$\nabla^{2} \varphi=-4 \pi \sum_{\alpha} e_{\alpha} \int f_{\alpha} d^{3} v$

In Eq. (1), $f_{\alpha 0}(v)$ is a spatially homogeneous zero order distribution function which we take to be a two-temperature Maxwellian,

$f_{\alpha 0}(v)=\left(\frac{m_{\alpha}}{2 \pi T_{\alpha \mathrm{n}}}\right)^{1 / 2} \frac{m_{\alpha}}{2 \pi T_{\alpha \perp}} e^{-\frac{m_{\alpha} v_{z}^{2}}{2 T_{\alpha \mathrm{n}}}} e^{-\frac{m_{\alpha}}{2 T_{\alpha \perp}}\left(v_{x}^{2}+v_{y}^{2}\right)}$

where the usual symbols $\mathrm{n}$ and $\perp$ are referred to the direction of the static magnetic field $B_{0}$. The surface wave has a phasor $\propto e^{i k_{y} y+i z_{z} z-i \omega t}$

Solving the set of equations, Eqs. (1) and (2) in a plasma slab requires a kinematic boundary condition on $f_{\alpha}$ at the interfaces $x=0$ and $x=L$. We adopt here the specular reflection boundary condition according to which the plasma particles undergo a mirror reflection such that

$$
\begin{aligned}
& f_{\alpha}\left(x=0, y, z, v_{x}, v_{y}, v_{z}, t\right)=f_{\alpha}\left(x=0, y, z,-v_{x}, v_{y}, v_{z}, t\right) \\
& f_{\alpha}\left(x=L, y, z, v_{x}, v_{y}, v_{z}, t\right)=f_{\alpha}\left(x=L, y, z,-v_{x}, v_{y}, v_{z}, t\right)
\end{aligned}
$$

We Fourier-transform Eqs. (1) and (2) with respect to the variables $y$ and $z$ (coordinates which do not contain the discontinuity) and $t$. Our convention of the Fouriertransform is

$$
\begin{aligned}
& f(k, v, \omega)=\int_{-\infty}^{\infty} d^{3} r \int_{-\infty}^{\infty} d t e^{-i k \cdot r+i \omega t} f(r, v, t) \\
& f(r, v, t)=\left(\frac{1}{2 \pi}\right)^{4} \int_{-\infty}^{\infty} d^{3} k \int_{-\infty}^{\infty} d \omega e^{i k \cdot r-i \omega t} f(k, v, \omega)
\end{aligned}
$$

In the following, we designate the Fourier-transformed quantities by explicitly writing the arguments. For example, $\varphi\left(k_{x}, k_{y}, k_{z}, \omega\right)$ and $\varphi\left(x, k_{y}, k_{z}, \omega\right)$ have different dimensions. We then obtain from Eqs. (1) and (2)

$v_{x} \frac{\partial}{\partial x} f_{\alpha}\left(x, k_{y}, k_{z}, v, \omega\right)-i\left(\omega-k_{y} v_{y}-k_{z} v_{z}\right) f_{\alpha}+\omega_{c \alpha}\left(v_{y} \frac{\partial}{\partial v_{x}}-v_{x} \frac{\partial}{\partial v_{y}}\right) f_{\alpha}=$

$\frac{e_{\alpha}}{m_{\alpha}}\left(\frac{\partial f_{\alpha 0}}{\partial v_{x}} \frac{\partial}{\partial x}+i k_{y} \frac{\partial f_{\alpha 0}}{\partial v_{y}}+i k_{z} \frac{\partial f_{\alpha 0}}{\partial v_{z}}\right) \varphi\left(x, k_{y}, k_{z}, \omega\right)$

where $\omega_{c \alpha}=\frac{e_{\alpha} B_{0}}{m_{\alpha} c}$. $\frac{\partial^{2} \varphi}{\partial x^{2}}-\left(k_{y}^{2}+k_{z}^{2}\right) \varphi\left(x, k_{y}, k_{z}, \omega\right)=-4 \pi \sum_{\alpha} e_{\alpha} \int d^{3} v f_{\alpha}\left(x, k_{y}, k_{z}, v, \omega\right)$

The specular reflection conditions, Eqs. (4) and (5), now take the forms in which the spatial coordinates $y, z$, and time $t$ are replaced by the Fourier variables $k_{y}, k_{z}$, and $\omega$, respectively. Equation (4) is automatically satisfied if $f_{\alpha}\left(x, v_{x}\right)=f_{\alpha}\left(-x,-v_{x}\right)$, i.e., if Eq. (6) is invariant under the reflections $\left(x \rightarrow-x, v_{x} \rightarrow-v_{x}\right)$. Here we should take into consideration the inherent symmetry of $f_{\alpha}$ in regard to the variables $\left(x, k_{y}, v_{x}, v_{y}\right)$

$f_{\alpha}\left(x, k_{y}, k_{z}, v_{x}, v_{y}, v_{z}, \omega\right)=f_{\alpha}\left(-x,-k_{y}, k_{z},-v_{x},-v_{y}, v_{z}, \omega\right)$

In regard to the above symmetry, we look at Eq. (15) which is the solution of Eq. (6) in terms of the Fourier variables: $f_{\alpha}$ is an even function with respect to simultaneous reflections of $v_{x}$ and $v_{y}$, and also an even function with respect to simultaneous reflections of $k_{x}$ and $k_{y}$, because $f_{\alpha}$ is a function of $v_{\perp}=\sqrt{v_{x}^{2}+v_{y}^{2}}$, $\phi=\tan ^{-1} \frac{v_{y}}{v_{x}}$, and $k_{\perp}=\sqrt{k_{x}^{2}+k_{y}^{2}}, \theta=\tan ^{-1} \frac{k_{y}}{k_{x}}$. In view of this symmetry, the specular reflection condition in Eq. (4) should read

$f_{\alpha}\left(x=0, k_{y}, k_{z}, v_{x}, v_{y}, v_{z}, \omega\right)=f_{\alpha}\left(x=0,-k_{y}, k_{z},-v_{x},-v_{y}, v_{z}, \omega\right)$

Equation (8) is automatically satisfied if Eq. (6) is invariant under the reflections $\left(x \rightarrow-x, v_{x} \rightarrow-v_{x}, k_{y} \rightarrow-k_{y}, v_{y} \rightarrow-v_{y}\right)$. By inspection, it is immediately seen that the electric potential $\varphi$ (defined in the plasma region $x>0$ should be extended into $x<0$ in the following way:

$\varphi\left(x, k_{y}\right)=\varphi\left(-x,-k_{y}\right)$

To enforce Eq. (5), we note that Eq. (6) is invariant under the reflections $\left(x \rightarrow 2 L-x, v_{x} \rightarrow-v_{x}, k_{y} \rightarrow-k_{y}, v_{y} \rightarrow-v_{y}\right)$, provided the potential is extended as

$\varphi\left(x, k_{y}\right)=\varphi\left(2 L-x,-k_{y}\right)$

The consequences of the extensions made in Eqs. (9) and (10) should be carefully collected in the Fourier transform of Eq. (7). We assume that $\varphi\left(x, k_{y}\right)$ is an even function with respect to both $x$ and $k_{y}$.

It is sufficient to consider the $x$-dependence of $\varphi\left(x, k_{y}\right)$ in carrying out the Fourier transform of Eq. (7) over the entire region $-\infty<x<\infty$. The function $\varphi(x)$ which has the properties

$\varphi(x)=\varphi(-x)$ and $\varphi(x)=\varphi(2 L-x)$ 
has discontinuities of $\frac{\partial \varphi}{\partial x}$ at $x=0,2 L, 4 L, \cdots$ of the same value. The derivative $\frac{\partial \varphi}{\partial x}$ has another value of jump at $x=L, 3 L, 5 L, \cdots$. Thus we have for the Fourier transform (with respect to $x$ ) of Eq. (7),

$k^{2} \varphi(k, \omega)-4 \pi \sum_{\alpha} e_{\alpha} \int f_{\alpha}(k, v, \omega) d^{3} v$

$=A\left(k_{y}, k_{z}, \omega\right)\left(\frac{1}{2}+\cos 2 k_{x} L+\cos 4 k_{x} L+\cdots\right)$

$+B\left(k_{y}, k_{z}, \omega\right)\left(\cos k_{x} L+\cos 3 k_{x} L+\cdots\right)$

where $A=\left.4 \frac{\partial \varphi}{\partial x}\right|_{x=0^{-}}$and $B=\left.4 \frac{\partial \varphi}{\partial x}\right|_{x=L^{-}}$. The details of similar algebra leading to Eq. (12) are provided in the earlier work [17].

\section{DISPERSION RELATION}

We introduce cylindrical coordinates in the velocity space such that

$v_{x}=v_{\perp} \cos \phi, \quad v_{y}=v_{\perp} \sin \phi$

Now Eq. (6) is Fourier-transformed to yield

$$
\begin{aligned}
& \frac{\partial}{\partial \phi} f_{\alpha}(k, \omega, v)+i \frac{\omega-k \cdot v}{\omega_{c \alpha}} f_{\alpha} \\
& =-\frac{i e_{\alpha} \varphi(k, \omega)}{m_{\alpha} \omega_{c \alpha}}\left[\frac{\partial f_{\alpha 0}}{\partial v_{\perp}}\left(k_{x} \cos \phi+k_{y} \sin \phi\right)+k_{z} \frac{\partial f_{\alpha 0}}{\partial v_{z}}\right]
\end{aligned}
$$

Equation (13) is a first order differential equation with respect to $\phi$. Integrating this gives

$$
\begin{aligned}
& f_{\alpha}\left(\phi, v_{\perp}, v_{z}, k, \omega\right)=\frac{-i e_{\alpha} \varphi(k, \omega)}{m_{\alpha} \omega_{c \alpha}} e^{i \Phi_{\alpha}(\phi)} \int_{ \pm \infty}^{\phi} d \phi^{\prime}\left[k_{\perp} \frac{\partial f_{\alpha 0}}{\partial v_{\perp}} \cos \left(\phi^{\prime}-\theta\right)+\right. \\
& \left.+k_{z} \frac{\partial f_{\alpha 0}}{\partial v_{z}}\right] e^{-i \Phi_{\alpha}\left(\phi^{\prime}\right)}
\end{aligned}
$$

where

$$
\begin{aligned}
k_{\perp} & =\sqrt{k_{x}^{2}+k_{y}^{2}}, k_{x}=k_{\perp} \cos \theta, k_{y}=k_{\perp} \sin \theta, \Phi_{\alpha}(\phi) \\
& =\frac{\left(k_{z} v_{z}-\omega\right) \phi+k_{\perp} v_{\perp} \sin (\phi-\theta)}{\omega_{c \alpha}}
\end{aligned}
$$

and the $+(-)$ sign at the lower limit of the integral corresponds to ion (electron). Then the integrated term vanishes at the lower limit for either species (here we assume that $\omega$ has a small positive imaginary part, in conjunction with our definition of the Fourier transform, to be consistent with the causality). The integral in Eq. (14) is carried out with the aid of the well-known Bessel function identity

$$
\begin{aligned}
& e^{i a \sin \phi}=\sum_{n=-\infty}^{\infty} J_{n}(a) e^{i n \phi}: \\
& f_{\alpha}(k, v, \omega)=-\frac{e_{\alpha} \varphi(k, \omega)}{m_{\alpha}} \sum_{n=-\infty}^{\infty} \sum_{l=-\infty}^{\infty}\left(\frac{\partial f_{\alpha 0}}{\partial v_{\perp}} \frac{l \omega_{c \alpha}}{v_{\perp}}+k_{z} \frac{\partial f_{\alpha 0}}{\partial v_{z}}\right)
\end{aligned}
$$

$\times J_{n}\left(a_{\alpha}\right) J_{l}\left(a_{\alpha}\right) \frac{e^{i(l-n) \theta} e^{i(n-l) \phi}}{\omega-k_{z} v_{z}-l \omega_{c \alpha}}$

where $a_{\alpha}=\frac{k_{\perp} v_{\perp}}{\omega_{c \alpha}}$. Substituting Eq. (15) into Eq. (12), we can obtain

$\varphi(k, \omega)=\frac{R\left(k_{y}, k_{z}, \omega\right)}{k^{2} \varepsilon_{L}}$

where $R\left(k_{y}, k_{z}, \omega\right)$ is the right hand side of Eq. (12) and

$\varepsilon_{L}=1+\sum_{\alpha} \frac{\omega_{p \alpha}^{2}}{k^{2}} \sum_{n=-\infty}^{\infty} \int d^{3} v\left(\frac{n \omega_{c \alpha}}{v_{\perp}} \frac{\partial f_{\alpha 0}}{\partial v_{\perp}}+k_{z} \frac{\partial f_{\alpha 0}}{\partial v_{z}}\right) \frac{J_{n}^{2}\left(a_{\alpha}\right)}{\omega-k_{z} v_{z}-n \omega_{c \alpha}}$

where $\omega_{p \alpha}$ is the plasma frequency. In obtaining Eq. (17), we used the fact that only the terms of $n=l$ survive in the $\int d \phi$-integral of Eq. (15). In an infinite plasma, $R=0$ and $\varepsilon_{L}=0$ gives the dispersion relation of the electrostatic waves. $\varepsilon_{L}$ is the dielectric constant of the magnetized plasma under consideration. From Eq. (16), one obtains the electric field components,

$E_{j}(k, \omega)=-i \frac{k_{j} R}{k^{2} \varepsilon_{L}} \quad(j=x, y, z)$

We need the normal component of the electric displacement.

$D_{x}(k, \omega)=E_{x}(k, \omega)+\frac{4 \pi i}{\omega} \sum_{\alpha} e_{\alpha} \int v_{x} f_{\alpha}(k, v, \omega) d^{3} v$

Using Eq. (15) in the above equation, with the aid of $\varphi=i \frac{E_{x}}{k_{x}}$, gives

$D_{x}=E_{x}+\frac{E_{x}}{\omega k_{x}} \sum_{\alpha} \frac{\omega_{p \alpha}^{2}}{2} \int d^{3} v v_{\perp} \sum_{n} \sum_{l}\left(\frac{\partial f_{\alpha 0}}{\partial v_{\perp}} \frac{l \omega_{c \alpha}}{v_{\perp}}+k_{z} \frac{\partial f_{\alpha 0}}{\partial v_{z}}\right)$

$\times \frac{J_{n} J_{l} e^{i(l-n) \theta}}{\omega-k_{z} v_{z}-l \omega_{c \alpha}}\left[e^{i(n-l+1) \phi}+e^{i(n-l-1) \phi}\right]$

In the above integral, only the terms of $n=l \pm 1$ survive in the $d \phi$-integral, and we obtain

$$
D_{x}(k, \omega)=\varepsilon_{x}(k, \omega) E_{x}(k, \omega)=-i \frac{k_{x} \varepsilon_{x}}{k^{2} \varepsilon_{L}} R\left(k_{y}, k_{z}, \omega\right)
$$

where

$$
\begin{aligned}
& \varepsilon_{x}=1+\sum_{\alpha} \frac{\omega_{p \alpha}^{2}}{\omega k_{\perp}} \int d^{3} v v_{\perp} \sum_{l=-\infty}^{\infty}\left(\frac{\partial f_{\alpha 0}}{\partial v_{\perp}} \frac{l \omega_{c \alpha}}{v_{\perp}}+k_{z} \frac{\partial f_{\alpha 0}}{\partial v_{z}}\right) \\
& \times \frac{1}{\omega-k_{z} v_{z}-l \omega_{c \alpha}}\left(\frac{l J_{l}^{2}}{a_{\alpha}}+i \frac{k_{y}}{k_{x}} J_{l} J_{l^{\prime}}\right)
\end{aligned}
$$

where $J_{l^{\prime}}=\frac{d J_{l}\left(a_{\alpha}\right)}{d a_{\alpha}}$, and we used the Bessel function relations 
$J_{l-1}+J_{l+1}=\frac{2 l}{a} J_{l}(a), \quad J_{l-1}-J_{l+1}=2 J_{l^{\prime}}$

The vacuum equations $\nabla^{2} \varphi=0$ is solved for $x<0$ and $x>L$

$x<0: \varphi\left(x, k_{y}, k_{z}, \omega\right)=F_{1} e^{\kappa x+i k_{y} y+i k_{z}^{z-i \omega t}}$

$x>L: \varphi\left(x, k_{y}, k_{z}, \omega\right)=F_{2} e^{-\kappa x+i k_{y} y+i k_{z} z-i \omega t}$

where $F_{1}$ and $F_{2}$ are arbitrary constants, and

$\kappa=\sqrt{k_{y}^{2}+k_{z}^{2}}$

The electric components are given by

$x<0: E_{x}=-\kappa F_{1} e^{\kappa x}, E_{y}=-i k_{y} F_{1} e^{\kappa x}, E_{z}=-i k_{z} F_{1} e^{\kappa x}$

$x>L: E_{x}=\kappa F_{2} e^{-\kappa x}, E_{y}=-i k_{y} F_{2} e^{-\kappa x}, E_{z}=-i k_{z} F_{2} e^{-\kappa x}$

where we omitted the phasor $e^{i k_{y} y+i k_{z} z-i \omega t}$.

The boundary conditions at the interface $x=0$ and $x=L$ are the continuity of $E_{y}$ (or $E_{z}$ ) and $D_{x}$. The conditions on $E_{z}$ are redundant to the conditions on $E_{y}$. To reinstate the $x$-dependance, the Fourier inversion integral should be performed on Eqs. (18) and (21). Let us first consider the integral:

$E_{y}\left(k_{y}, k_{z}, \omega, x\right)=-i k_{y} \int_{-\infty}^{\infty} \frac{d k_{x} e^{i k_{x} x}}{k^{2} \varepsilon_{L}}\left[A\left(\frac{1}{2}+\cos 2 k_{x} L+\cos 4 k_{x} L+\cdots\right)\right.$

$\left.+B\left(\cos k_{x} L+\cos 3 k_{x} L+\cos 5 k_{x} L+\cdots\right)\right]$

It is important to do the integral of the series term by term. Here we have

$\int_{-\infty}^{\infty} d k_{x} \frac{e^{i x k_{x}}}{k^{2} \varepsilon_{L}} \cos \left(n k_{x} L\right)=\frac{1}{2} \int_{-\infty}^{\infty} d k_{x} \frac{e^{i x k_{x}}}{k^{2} \varepsilon_{L}}\left(e^{i n k_{x} L}+e^{-i n k_{x} L}\right) \quad(n=1,2,3, \cdots)$

In the second integral, we make a change of variable $k_{x} \rightarrow-k_{x}$ and utilize that $\varepsilon_{L}$ is an even function of $k_{x}$. Then we have

$\int_{-\infty}^{\infty} d k_{x} \frac{e^{i x k_{x}}}{k^{2} \varepsilon_{L}} \cos \left(n k_{x} L\right)=\int_{-\infty}^{\infty} d k_{x} \frac{e^{i k_{x} n L}}{k^{2} \varepsilon_{L}} \cos \left(k_{x} x\right)$

Using Eq. (28) in Eq. (27) gives

$$
\begin{aligned}
& E_{y}\left(k_{y}, k_{z}, \omega, x=0\right)=-i k_{y} \int_{-\infty}^{\infty} \frac{d k_{x}}{k^{2} \varepsilon_{L}}\left[A\left(\frac{1}{2}+e^{2 i k_{x} L}+e^{4 i k_{x} L}+\cdots\right)\right. \\
& \left.+B\left(e^{i k_{x} L}+e^{3 i k_{x} L}+e^{5 i k_{x} L}+\cdots\right)\right] \\
& E_{y}\left(k_{y}, k_{z}, \omega, x=L\right)=-i k_{y} \int_{-\infty}^{\infty} d k_{x}\left[A \left(\frac{1}{2} e^{i L k_{x}}\right.\right. \\
& +\operatorname{cosk}_{x} L\left(e^{2 i k_{x} L}+e^{4 i k_{x} L}+\cdots\right) \\
& \left.+B \cos k_{x} L\left(e^{i k_{x} L}+e^{3 i k_{x} L}+e^{5 i k_{x} L}+\cdots\right)\right]
\end{aligned}
$$

Writing the cosine functions in terms of exponential functions, one can easily obtain

$$
\begin{aligned}
& E_{y}\left(k_{y}, k_{z}, \omega, x=L\right)=-i k_{y} \int_{-\infty}^{\infty} \frac{d k_{x}}{k^{2} \varepsilon_{L}}\left[B\left(\frac{1}{2}+e^{2 i k_{x} L}+e^{4 i k_{x} L}+\cdots\right)\right. \\
& \left.+A\left(e^{i k_{x} L}+e^{3 i k_{x} L}+e^{5 i k_{x} L}+\cdots\right)\right]
\end{aligned}
$$

Next we calculate the integral

$D_{x}\left(k_{y}, k_{z}, \omega, x\right)=-i \int_{-\infty}^{\infty} d k_{x} e^{i k_{x} x} \frac{k_{x} \varepsilon_{x}}{k^{2} \varepsilon_{L}}\left[A\left(\frac{1}{2}+\cos 2 k_{x} L+\cos 4 k_{x} L+\cdots\right)\right.$

$\left.+B\left(\cos k_{x} L+\cos 3 k_{x} L+\cos 5 k_{x} L+\cdots\right)\right]$

Here we consider a typical term

$$
\int_{-\infty}^{\infty} d k_{x} e^{i k_{x} x} \frac{k_{x} \varepsilon_{x}}{k^{2} \varepsilon_{L}} \cos \left(n k_{x} L\right) \quad(n=1,2,3, \cdots)
$$

where $\varepsilon_{x}$ consists of two parts, the even function part and the odd function part, with respect to $k_{y}: \varepsilon_{x}=\varepsilon^{E}+\varepsilon^{O}$ with

$$
\begin{aligned}
& \varepsilon^{E}=1+\sum_{\alpha} \frac{\omega_{p \alpha}^{2}}{\omega k_{\perp}} \int d^{3} v v_{\perp} \sum_{l=-\infty}^{\infty}\left(\frac{\partial f_{\alpha 0}}{\partial v_{\perp}} \frac{l \omega_{c \alpha}}{v_{\perp}}+k_{z} \frac{\partial f_{\alpha 0}}{\partial v_{z}}\right) \frac{l J_{l}^{2} / a_{\alpha}}{\omega-k_{z} v_{z}-l \omega_{c \alpha}} \\
& \varepsilon^{o}=\sum_{\alpha} \frac{\omega_{p \alpha}^{2}}{\omega k_{\perp}} \int d^{3} v v_{\perp} \sum_{l=-\infty}^{\infty}\left(\frac{\partial f_{\alpha 0}}{\partial v_{\perp}} \frac{l \omega_{c \alpha}}{v_{\perp}}+k_{z} \frac{\partial f_{\alpha 0}}{\partial v_{z}}\right) \frac{i k_{y} J_{l} J_{l^{\prime}} / k_{x}}{\omega-k_{z} v_{z}-l \omega_{c \alpha}}
\end{aligned}
$$

Writing in (32) the cosine function in terms of exponential functions and making change of variable $k_{x} \rightarrow-k_{x}$ as before, we have

$\int_{-\infty}^{\infty} d k_{x} e^{i k_{x} x} \frac{k_{x} \varepsilon_{x}}{k^{2} \varepsilon_{L}} \cos \left(n k_{x} L\right)=\int_{-\infty}^{\infty} d k_{x} e^{i x_{x}{ }^{n L}} \frac{k_{x}}{k^{2} \varepsilon_{L}}\left(\varepsilon^{E} i \sin k_{x} x+\varepsilon^{0} \cos k_{x} x\right)$

Using Eq. (35) in Eq. (31) gives after some algebra

$$
\begin{aligned}
& D_{x}\left(k_{y}, k_{z}, \omega, x=0\right) \\
& =-i A \int_{-\infty}^{\infty} d k_{x} \frac{k_{x}}{k^{2} \varepsilon_{L}}\left[\frac{+1}{2} \varepsilon^{E}+\varepsilon^{O}\left(\frac{1}{2}+e^{2 i k_{x} L}+e^{4 i k_{x} L}+\cdots\right)\right] \\
& \left.-i B \int_{-\infty}^{\infty} d k_{x} \frac{k_{x}}{k^{2} \varepsilon_{L}} \varepsilon^{O}\left(e^{i k_{x} L}+e^{3 i k_{x} L}+e^{5 i k_{x} L}+\cdots\right)\right] \\
& D_{x}\left(k_{y}, k_{z}, \omega, x=L\right) \\
& =-i B \int_{-\infty}^{\infty} d k_{x} \frac{k_{x}}{k^{2} \varepsilon_{L}}\left[\frac{-1}{2} \varepsilon^{E}+\varepsilon^{O}\left(\frac{1}{2}+e^{2 i k_{x} L}+e^{4 i k_{x} L}+\cdots\right)\right] \\
& \left.-i A \int_{-\infty}^{\infty} d k_{x} \frac{k_{x}}{k^{2} \varepsilon_{L}} \varepsilon^{O}\left(e^{i k_{x} L}+e^{3 i k_{x} L}+e^{5 i k_{x} L}+\cdots\right)\right]
\end{aligned}
$$

Enforcing the boundary conditions on $x=0$ and $x=L$ give four algebraic equations for four undetermined constants, A, B, $F_{1}$, and $F_{2}$. The solvability condition yields the relation

$$
\mu^{2}-\gamma^{2}=\beta^{2}-\delta^{2}
$$

where 


$$
\begin{aligned}
& \mu=\int \frac{d k_{x}}{k^{2} \varepsilon_{L}}\left(\frac{1}{2} k_{x} \varepsilon^{E}+i \kappa\left[\frac{1}{2}+e^{2 i k_{x} L}+e^{4 i k_{x} L}+\cdots\right]\right) \\
& \beta=\int \frac{d k_{x} k_{x}}{k^{2} \varepsilon_{L}} \varepsilon^{O}\left(\frac{1}{2}+e^{2 i k_{x} L}+e^{4 i k_{x} L}+\cdots\right) \\
& \gamma=\int \frac{i \kappa d k_{x}}{k^{2} \varepsilon_{L}}\left(e^{i k_{x} L}+e^{3 i k_{x} L}+e^{5 i k_{x} L}+\cdots\right) \\
& \delta=\int \frac{d k_{x} k_{x}}{k^{2} \varepsilon_{L}} \varepsilon^{O}\left(e^{i k_{x} L}+e^{3 i k_{x} L}+e^{5 i k_{x} L}+\cdots\right)
\end{aligned}
$$

Here obtaining solutions of the dispersion relation, Eq. (38), is facilitated by the observation that it is intact if we replace $k_{y} \rightarrow-k_{y}$. This means that if $\xi\left(k_{y}\right)$ is a solution of Eq. (38), $\xi\left(-k_{y}\right)$ also solves Eq. (38). To take advantage of this even parity in $k_{y}$ of Eq. (38), we write it as

$$
(\beta+\mu)(\beta-\mu)=(\delta+\gamma)(\delta-\gamma)
$$

We seek the solutions of Eq. (43) by equating as

$\beta+\mu=\delta+\gamma$

It is immediately seen that Eq. (44) indeed solves Eq. (43) because changing $k_{y} \rightarrow-k_{y}$ in Eq. (44) yields the relation $-\beta+\mu=-\delta+\gamma$, which is the remaining relation to be satisfied in Eq. (43). The second reduction of the dispersion relation, Eq. (38), is obtained by writing it as

$(\beta+\mu)(\beta-\mu)=(-1)(\delta+\gamma) \cdot(-1)(\delta-\gamma)$

We can see that the relation

$\beta-\mu=-(\delta-\gamma)=\gamma-\delta$

contains another set of solutions of Eq. (38) because the rest of relation of Eq. (45) is automatically satisfied. In summary, we have two independent reductions of the dispersion relation, Eq. (38)

$\mu \pm \gamma=\delta \pm \beta$

It turns out that the lower (upper) sign corresponds to the symmetric (anti-symmetric) mode. The solutions obtained from Eq. (47) by replacing $k_{y} \rightarrow-k_{y}$ are also legitimate solutions of the slab dispersion relation.

Using the expressions in Eqs. (39)-(42), the dispersion relation is written

$\int_{-\infty}^{\infty} \frac{d k_{x}}{k^{2} \varepsilon_{L}}\left[\begin{array}{c}\frac{k_{x} \varepsilon^{E}}{2}+\left(i \kappa+k_{x} \varepsilon^{O}\right)\left(\frac{1}{2} \pm e^{i L k_{x}}\right. \\ \left.+e^{2 i L k_{x}} \pm e^{3 i L k_{x}}+e^{4 i L k_{x}} \pm \cdots\right)\end{array}\right]=0$

Clearly the series in the parentheses converge by picking the poles of $k^{2} \varepsilon_{L}=0$ in the upper-half $k_{x}$-plane and the exponential terms vanish when $L \rightarrow \infty$. We can sum the series formally to make the expression compact. It can be easily shown that we have the following expressions for the series,

$$
\begin{aligned}
& e^{i L k_{x}}+e^{2 i L k_{x}}+e^{3 i L k_{x}}+\cdots=\frac{e^{i L k_{x}}}{1-e^{i L k_{x}}} \\
& e^{i L k_{x}}+e^{3 i L k_{x}}+e^{5 i L k_{x}}+\cdots=\frac{e^{i L k_{x}}}{1-e^{2 i L k_{x}}} \\
& e^{2 i L k_{x}}+e^{4 i L k_{x}}+e^{6 i L k_{x}}+\cdots=\frac{e^{2 i L k_{x}}}{1-e^{2 i L k_{x}}}
\end{aligned}
$$

Thus we obtain for the dispersion relation in Eq. (47)

$\int_{-\infty}^{\infty} \frac{d k_{x}}{k^{2} \varepsilon_{L}}\left[k_{x} \varepsilon^{E}+\left(i \kappa+k_{x} \varepsilon^{O}\right) \frac{1 \pm e^{i k_{x} L}}{1 \mp e^{i k_{x} L}}\right]=0$

where the apparent singularities associated with the denominator $1 \mp e^{i k_{x} L}=0$ should be simply disregarded. If $k_{y}=0$, we have $\varepsilon^{O}=0$ and Eq. (48) agrees with the result obtained in earlier work (Eq. (55) in Ref. [18]). If $L \rightarrow \infty$, the factor $e^{i k_{x} L}$ vanishes (by piking the poles in the upperhalf $k_{x}$-plane), and Eq. (48) becomes

$\int d k_{x} \frac{k_{x} \varepsilon_{x}+i \kappa}{k^{2} \varepsilon_{L}}=0$

Equation (49) recovers the electrostatic dispersion relation for a semi-infinite plasma obtained in Ref. [18] (Eq. (37) therein).

To further check the correctness of Eq. (48), its cold plasma limit will be considered. We put

$f_{\alpha 0}(v)=\frac{\delta\left(v_{\perp}\right)}{v_{\perp}} \delta\left(v_{z}\right) \delta(\phi)$

into Eq. (17) for $\varepsilon_{L}$. We integrate by parts each term, and use

$$
J_{n}(0)=\delta_{n, 0}, \frac{2 n}{a} J_{n}(a) J_{n^{\prime}}(a) \rightarrow \frac{1}{2}\left(\delta_{n, 1}-\delta_{n,-1}\right)
$$

in the limit of $a \rightarrow 0$, to obtain

$\varepsilon_{L}^{\text {cold }}=1-\sum_{\alpha} \frac{\omega_{p \alpha}^{2}}{k^{2}}\left(\frac{k_{z}^{2}}{\omega^{2}}+\frac{k_{\perp}^{2}}{\omega^{2}-\omega_{c \alpha}^{2}}\right)$

The cold plasma limit of $\varepsilon_{x}$ (Eq. (22)) can be calculated similarly. It can be easily shown that the terms involving $\frac{\partial f_{\alpha 0}}{\partial v_{z}}$ vanish in the limit of $a_{\alpha} \rightarrow 0$. The first term of the velocity integral containing $J_{l}^{2}$ in the rest of Eq. (22) yields

$$
-\sum_{\alpha} \frac{\omega_{p \alpha}^{2}}{\omega^{2}-\omega_{c \alpha}^{2}}
$$

The second term involving $J_{l} J_{l^{\prime}}$ becomes after integration by parts with respect to $v_{\perp}$

$$
-i \sum_{\alpha} \frac{\omega_{p \alpha}^{2}}{\omega k_{\perp}} \frac{k_{y}}{k_{x}} \sum_{l} \frac{l \omega_{c \alpha}}{\omega-l \omega_{c \alpha}} \int d v_{\perp} \frac{\delta\left(v_{\perp}\right)}{v_{\perp}} \frac{d}{d v_{\perp}}\left[v_{\perp} J_{l} J_{l^{\prime}}\right]
$$


Denoting the $\int d v_{\perp}-$ integral by $I_{\alpha}$, we have

$$
\begin{aligned}
I_{\alpha} & =\int d a_{\alpha} \frac{1}{a_{\alpha}} \delta\left(a_{\alpha} \frac{\omega_{c \alpha}}{k_{\perp}}\right) \frac{d}{d a_{\alpha}}\left[a_{\alpha} J_{l} J_{l^{\prime}}\right] \\
& =\frac{k_{\perp}}{\omega_{c \alpha}} \lim _{a_{\alpha} \rightarrow 0}\left[\frac{J_{l} J_{l^{\prime}}}{a_{\alpha}}+J_{l^{\prime}} J_{l^{\prime}}+J_{l} J_{l}^{\prime \prime}\right]
\end{aligned}
$$

Upon using the asymptotic values of the Bessel functions, the last term involving $J_{l}^{\prime \prime}$ vanishes. Using Eq. (42) and $J_{l^{\prime}} \rightarrow \frac{1}{2}\left(\delta_{l, 1}-\delta_{l,-1}\right)$, we obtain

$$
l I_{\alpha}=-\frac{k_{\perp}}{2 \omega_{c \alpha}}\left(\delta_{l, 1}-\delta_{l,-1}\right)
$$

Collecting the preceding results, we finally obtain for the cold plasma limit of $\varepsilon_{x}$

$\varepsilon_{x}^{\text {cold }}=1-\sum_{\alpha}\left(1-\frac{i k_{y}}{k_{x}} \frac{\omega_{c \alpha}}{\omega}\right) \frac{\omega_{p \alpha}^{2}}{\omega^{2}-\omega_{c \alpha}^{2}}$

$\varepsilon^{E}=1-\sum_{\alpha} \frac{\omega_{p \alpha}^{2}}{\omega^{2}-\omega_{c \alpha}^{2}} \equiv P$,

$\varepsilon^{O}=\frac{i k_{y}}{k_{x}} \sum_{\alpha} \frac{\omega_{c \alpha}}{\omega} \frac{\omega_{p \alpha}^{2}}{\omega^{2}-\omega_{c \alpha}^{2}} \equiv \frac{i k_{y}}{k_{x}} Q$

Substituting Eqs. (51) and (56) into Eq. (48) and carrying out the contour integrals, we can obtain the cold fluid limit of the electrostatic slab dispersion relation. The first term of Eq. (48) is calculated to obtain

$\int d k_{x} \frac{k_{x} \varepsilon^{E}}{k^{2} \varepsilon_{L}^{\text {cold }}}=\int d k_{x} \frac{P k_{x}}{P\left(k_{x}^{2}+\lambda^{2}\right)}=i \pi$

where

$\lambda=\sqrt{k_{y}^{2}+k_{z}^{2} \frac{C}{P}}, \quad C=1-\sum_{\alpha} \frac{\omega_{p \alpha}^{2}}{\omega^{2}}$

Similar calculation for the rest of the terms in Eq. (48) yields the slab dispersion relations

$P \lambda+\left(Q k_{y}+\kappa\right) \operatorname{coth}\left(\frac{\lambda L}{2}\right)=0, \quad($ anti - symmetric mode $)$

$P \lambda+\left(Q k_{y}+\kappa\right) \tanh \left(\frac{\lambda L}{2}\right)=0,($ symmetric mode $)$

If $L \rightarrow \infty$, the two modes coalesce to

$P \lambda+Q k_{y}+\kappa=0$

which is the cold plasma electrostatic dispersion relation in the magnetized semi-infinite plasma. Furthermore, if $k_{z}=0$, we have $\lambda=\kappa=k_{y}$, and the dispersion relation takes the form

$$
2+\sum_{\alpha} \frac{\omega_{p \alpha}^{2}}{\omega^{2}-\omega_{c \alpha}^{2}}\left(\frac{\omega_{c \alpha}}{\omega}-1\right)=0
$$

Equation (62) agrees with the dispersion relation obtained earlier from the cold fluid equations in Ref. [19] (Eq. (40) therein).

\section{FLUID TREATMENT}

We use the following two-fluid equations to obtain the plasma solutions of the electrostatic field for $0<x<L$ :

$-i \omega v_{\alpha}=-\frac{e_{\alpha}}{m_{\alpha}} \nabla \varphi+\omega_{c \alpha} v_{\alpha} \times z$

$-i \omega n_{\alpha}+N \nabla \cdot v_{\alpha}=0$

$\nabla^{2} \varphi=4 \pi e\left(n_{e}-n_{i}\right)$

where $\varphi=\varphi(\omega, r)$, and $\mathrm{N}$ is the equilibrium density of the plasma. One can obtain from the above equations

$v_{\alpha x}=\frac{i e_{\alpha}}{m_{\alpha}} \frac{\omega}{\omega_{c \alpha}^{2}-\omega^{2}}\left(\frac{\partial \varphi}{\partial x}+i \frac{\omega_{c \alpha}}{\omega} \frac{\partial \varphi}{\partial y}\right)$

$\frac{\partial^{2} \varphi}{\partial x^{2}}-\lambda^{2} \varphi=0$

where $\lambda$ is defined by Eq. (58). Solving the above equation gives

$\varphi(x, y, z, \omega)=\left(A_{1} e^{-\lambda x}+A_{2} e^{\lambda x}\right) e^{i k_{y} y+i k_{z} z-i \omega t}$

which in turn yields (omitting the phasor)

$$
\begin{aligned}
& E_{x}=\lambda\left(A_{1} e^{-\lambda x}-A_{2} e^{\lambda x}\right) \\
& E_{y}=-i k_{y}\left(A_{1} e^{-\lambda x}+A_{2} e^{\lambda x}\right) \\
& E_{z}=-i k_{z}\left(A_{1} e^{-\lambda x}+A_{2} e^{\lambda x}\right)
\end{aligned}
$$

where $A_{1}$ and $A_{2}$ are constants. The vacuum solutions can be written down as

$$
\begin{aligned}
& x<0: E_{x}=-\kappa F_{1} e^{\kappa x}, E_{y}=-i k_{y} F_{1} e^{\kappa x}, E_{z}=-i k_{z} F_{1} e^{\kappa x} \\
& x>L: E_{x}=\kappa F_{2} e^{-\kappa x}, E_{y}=-i k_{y} F_{2} e^{-\kappa x}, E_{z}=-i k_{z} F_{2} e^{-\kappa x}
\end{aligned}
$$

where $F_{1}$ and $F_{2}$ are constants.

We need the normal component of the electric displacement vector, $D_{x}$, in the plasma:

$D_{x}=E_{x}+\frac{4 \pi N i}{\omega} \sum_{\alpha} e_{\alpha} v_{\alpha x}$

Using Eqs. (66) and (68) in the above equation gives

$D_{x}=P E_{x}-i Q E_{y}=A_{1} e^{-\lambda x}\left(\lambda P-Q k_{y}\right)-A_{2} e^{\lambda x}\left(\lambda P+Q k_{y}\right)$

where $\mathrm{P}$ and $\mathrm{Q}$ are defined by Eqs. (56).

The continuity of $E_{y}$ at $x=0$ and $x=L$ yields

$A_{1}+A_{2}=F_{1}$

$A_{1} e^{-\lambda L}+A_{2} e^{\lambda L}=F_{2} e^{-\kappa L}$ 
The continuity of $D_{x}$ at $x=0$ and $x=L$ yields

$-\kappa F_{1}=A_{1}\left(P \lambda-Q k_{y}\right)-A_{2}\left(P \lambda+Q k_{y}\right)$

$\kappa F_{2} e^{-\kappa L}=A_{1} e^{-\lambda L}\left(P \lambda-Q k_{y}\right)-A_{2} e^{\lambda L}\left(P \lambda+Q k_{y}\right)$

The solvability condition of the above four equations gives

$e^{\frac{\lambda L}{2}}\left(P \lambda+\kappa+Q k_{y}\right) e^{\frac{\lambda L}{2}}\left(P \lambda+\kappa-Q k_{y}\right)=$

$e^{-\frac{\lambda L}{2}}\left(P \lambda-\kappa+Q k_{y}\right) e^{\frac{-\lambda L}{2}}\left(P \lambda-\kappa-Q k_{y}\right)$

which is the slab dispersion relation obtained from the fluid equations. Equation (80) has the even parity in $k_{y}$ : Eq. (80) is intact if we replace $k_{y} \rightarrow-k_{y}$. We scan obtain the solutions of Eq. (80) by equating as

$e^{\frac{\lambda L}{2}}\left(P \lambda+\kappa+Q k_{y}\right)=e^{-\frac{\lambda L}{2}}\left(P \lambda-\kappa-Q k_{y}\right)$

because the rest of Eq. (80) is nothing but the relation obtained by replacing $k_{y} \rightarrow-k_{y}$ in the above equation. Another independent relation is obtained by equating as

$$
e^{\frac{\lambda L}{2}}\left(P \lambda+\kappa+Q k_{y}\right)=-e^{-\frac{\lambda L}{2}}\left(P \lambda-\kappa-Q k_{y}\right)
$$

Equations (81) and (82) can be arranged into the forms of Eqs. (59) and (60), showing complete agreement with the cold fluid limit of the kinetic results.

\section{DISCUSSION}

The surface waves with phasor $\propto e^{i k_{z} z+i k_{y} y-i \omega t}$ propagating in a magnetized slab as well as on a magnetized semi-infinite plasma with $B_{0}=z B_{0}$ (directed parallel to the interface) have an interesting property with respect to the wavenumber $k_{y}$. Because of the even parity of $k_{y}$, replacement of $k_{y} \rightarrow-k_{y}$ in our dispersion relations yields another set of dispersion relation. For example,

$P \lambda-Q k_{y}+\kappa=0$,

which is obtained from Eq. (61) by $k_{y} \rightarrow-k_{y}$, is also a legitimate dispersion relation. This property generates a variety of waves as compared to waves of no-parity. The pair of waves with $\pm k_{y}$ does not mean the oppositely propagating waves because we have the component $k_{z}$.

Finally, although our work obtained kinetic slab dispersion relation in closed form, nonlinear development of surface wave is of great interest. In this regard, we should mention the work of Stenflo [20] which addresses the important nonlinear problem of surface waves in semiinfinite plasma as well as in a plasma slab. Also three-wave surface wave interactions in a bounded plasma are investigated therein.

\section{REFERENCES}

[1] Vukovic V. Surface waves in plasmas and solids. Singapore: World Scientific 1986.

[2] Halevi P. Spatial dispersion in solids and plasmas. Amsterdam: Elsevier 1992.

[3] Benova E, Ghanashev I, Zhelyazkov I. Theoretical study of a plasma column sustained by an electromagnetic surface wave in the dipolar mode. J Plasma Phys 1991; 45: 137-52.

[4] Aliev YM, Schlüter H, Shivarova A. Guided-wave-produced plasmas. Berlin: Springer 2000.

[5] Buti B. Advances in space plasma physics. Singapore: World Scientific 1985.

[6] Gradov OM, Stenflo L. Linear-theory of a cold bounded plasma. Phys Rep 1983; 94: 111-137.

[7] Kaufman RN. Propagation of surface waves along a plane boundary between two magnetoactive plasmas. Soviet Phys-Tech Phys 1972; 17: 587-91.

[8] Uberoi C, Rao UJ. Surface waves on the boundary of a magnetized plasma. Plasma Phys 1975; 17: 659-70.

[9] Lee HJ, Cho SH. Dispersion of surface waves propagating along planar interfaces between plasmas or plasma and free space. Plasma Phys Control Fusion 1995; 37: 989-1002.

[10] Ivanov ST, Nonaka S, Nikolaev NI. The spectrum of electromagnetic waves in a magnetized gaseous plasma layer. Part 1. Wave dispersion. J Plasma Phys 2001; 65: 273-89.

[11] Alexandrov AF, Bogdankevich LS, Rukhadze AA. Principles of plasma electrodynamics. Berlin: Springer 1984.

[12] Aleksandrov AF, Rukhadze AA. Kinetic theory of surface waves in a semibounded plasma flow. Tech Phys 2000; 45: 396-9 [Zhurnal Tekhnicheskol Fiziki 2000; 70: 14-7].

[13] Lee HJ. Comment on "Kinetic theory of surface waves in plasma jets” [Phys. Plasmas 9, 701 (2002)]. Phys Plasmas 2005; 12: 094701-4.

[14] Landau L. On the vibrations of the electronic plasma. J Phys 1946; $\mathrm{X}: 25-34$.

[15] Barr HC, Boyd TJM. Surface waves in hot plasmas. J Phys A 1972; 5: 1108-18.

[16] Lee MJ, Lee HJ. Surface waves in anisotropic Maxwellian plasmas. Phys Plasmas 2005; 12: 052104-6.

[17] Lee HJ, Lim YK. Kinetic theory of surface waves in a plasma slab. J Korean Phys Soc 2007; 50: 1056-61.

[18] Lee HJ, Kim CG. Kinetic Theory of Electrostatic Surface Waves in Magnetized Plasmas. J Korean Phys Soc 2009; 54: 85-93.

[19] Lee HJ. Electrostatic surface waves in a magnetized two-fluid plasma. Plasma Phys Control Fusion 1995; 37: 755-62.

[20] Stenflo L. Theory of nonlinear plasma surface waves. Phys Scrip 1996; T63: 59-62. 